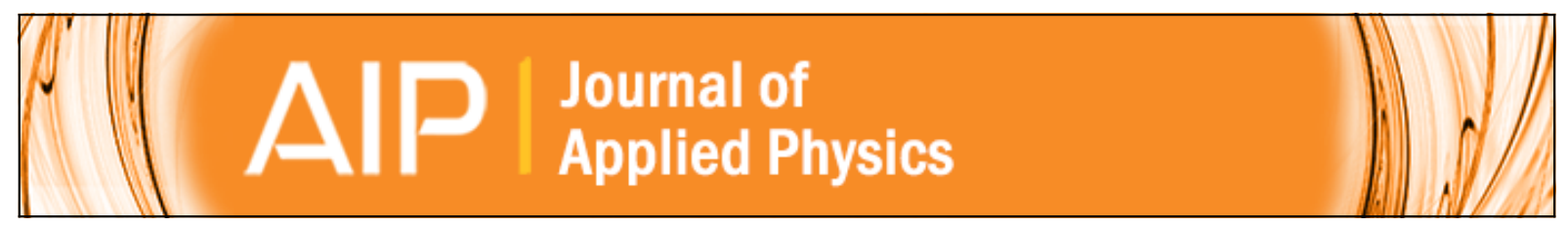

\title{
High quality Ti-implanted Si layers above the Mott limit
}

J. Olea, M. Toledano-Luque, D. Pastor, E. San-Andrés, I. Mártil, and G. González-Díaz

Citation: Journal of Applied Physics 107, 103524 (2010); doi: 10.1063/1.3391274

View online: http://dx.doi.org/10.1063/1.3391274

View Table of Contents: http://scitation.aip.org/content/aip/journal/jap/107/10?ver=pdfcov

Published by the AIP Publishing

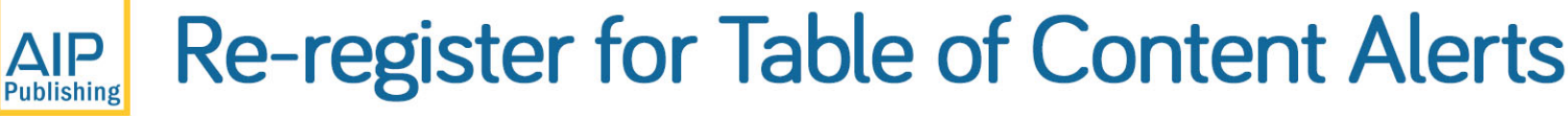

\section{Create a profile. \\ Sign up today!}




\title{
High quality Ti-implanted Si layers above the Mott limit
}

\author{
J. Olea, M. Toledano-Luque, D. Pastor, ${ }^{\text {a) }}$ E. San-Andrés, I. Mártil, and G. González-Díaz \\ Departamento de Física Aplicada III (Electricidad y Electrónica), Facultad de Ciencias Físicas, \\ Universidad Complutense de Madrid, E-28040 Madrid, Spain
}

(Received 21 December 2009; accepted 15 March 2010; published online 25 May 2010)

\begin{abstract}
In this paper, we present a detailed characterization of high quality layers of Si implanted with Ti at high doses. These layers are intended to the formation of an intermediate band (IB) solar cell. The main requirement to obtain an IB material is to reach an impurity concentration beyond the Mott limit, which is, in this case, much higher than the solid solubility limit. To overcome this limit we used the combination of ion implantation and pulsed-laser melting as nonequilibrium techniques. Time-of-flight secondary ion mass spectrometry measurements confirm that Ti concentration exceeds the theoretical Mott limit in the implanted layer, and glancing incidence x-ray diffraction and transmission electron microscopy measurements prove that good crystallinity can be achieved. Sheet resistance and Hall effect mobility show uncommon characteristics that can only been explained assuming the IB existence. (C) 2010 American Institute of Physics. [doi:10.1063/1.3391274]
\end{abstract}

\section{INTRODUCTION}

In the last decade, an enormous effort has been channeled toward the improvement of the efficiency in the solar cell technology. ${ }^{1}$ One of the most promising concepts is the intermediate band solar cell (IBSC), ${ }^{2}$ which has been leading the so-called third generation of photovoltaic technology. ${ }^{3}$ The IBSC is a forward-looking approach that can overcome the theoretical conversion limit for single junction solar cells. ${ }^{4}$ This new concept is based on the idea of placing a half-filled band of allowed states between the traditional valence and conduction bands that permits photons of energy lower than the semiconductor band gap to be absorbed, yielding a higher efficiency. Together with the optical transition from the valence band to the conduction band, two new transitions could show up: one from the valence band to the IB and another from the IB to the conduction band. In an IB material, an electron can be pumped from the valence band to the conduction band by one stage transition or by two stages transition. This ability could improve the absorption of incident photons, which translates in a higher efficiency conversion than the achieved in standard solar cells by means of a substantially higher short circuit current. ${ }^{5}$

Among other possibilities like quantum $\operatorname{dots}^{6}$ or highlymismatched alloys, ${ }^{7}$ ion implantation of deep level impurities can fulfill this task if the dose is high enough to exceed the Mott limit. ${ }^{8}$ In fact, if deep impurities are introduced with a concentration above this limit, deep-levels would overlap to form a continuous of allowed states, giving rise to the formation of an IB within the forbidden gap of the host semiconductor.

Surpassing the Mott limit implies usually a technological challenge because it is usually far away from the solubility limit, meaning that we are forced to use nonequilibrium techniques. Having this in mind, we have used the combination of two highly nonequilibrium techniques, namely, ion im-

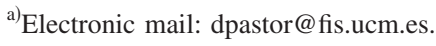

plantation and pulsed-laser melting (PLM) annealing. These techniques have been extensively used to design IB materials based in the highly mismatched alloys approach at the Lawrence Berkeley National Laboratory. ${ }^{9}$ With this approach, compound semiconductors with IB have been successfully described. ${ }^{10,11}$

Additionally, regarding "standard" semiconductors, theoretical $^{12}$ and experimental ${ }^{13}$ results suggest that the introduction of $\mathrm{Ti}$ in GaP (Ref. 14) and Si (Ref. 12) above the Mott limit could make these materials to behave as IB semiconductors. In the case of $\mathrm{Si}$, this is especially interesting because of the possibility to extend the highly mature $\mathrm{Si}$ solar cell technology to the fabrication of Si-based IBSCs. Moreover, the achievement of subband gap absorption in $\mathrm{Si}$ by means of $\mathrm{S}$ implantation and laser treated samples has been reported recently. ${ }^{15}$

Clearly, $\mathrm{Si}$ is not the optimum semiconductor from a point of view of the yield, but any increase in the present value of the $\mathrm{Si}$ based solar cells efficiency would mean a very important contribution to the photovoltaic industry.

The very high concentration of impurities required to obtain an IB could produce an important distortion on the structural characteristics of the host $\mathrm{Si}$. As a consequence, the degree of crystalline recovery after the implantation of such amount of impurities and/or the presence of secondary phase in the "new" semiconductor are issues that should be correctly addressed in order to assess the IB material as candidate for IBSC.

In a recent paper, ${ }^{16}$ we have analyzed the electrical transport properties of the $\mathrm{Ti}$ implanted layers on $n$-type $\mathrm{Si}$ samples. Sheet resistance could only be explained on the basis of the IB formation. IB position inside the Si gap and carrier concentration in this band could be deduced using the ATLAS simulation code.

In the present paper, we have performed a detailed structural analysis of the same samples used in a previous paper, ${ }^{16}$ showing excellent structural characteristics. 


\section{EXPERIMENTAL}

$n$-type silicon (111) samples with a thickness of $300 \mu \mathrm{m}$ $\left(\mu=1500 \mathrm{~cm}^{2} / \mathrm{V} \mathrm{s} ; n=2.2 \times 10^{13} \mathrm{~cm}^{-3}\right.$ at room temperature) were implanted in an IBS refurbished VARIAN CF3000 ion implanter at $30 \mathrm{keV}$ with $\mathrm{Ti}$ at high doses $\left(10^{15}\right.$, $5 \times 10^{15}, 10^{16}$, and $\left.5 \times 10^{16} \mathrm{~cm}^{-2}\right)$. After implantation, samples were PLM annealed at $0.8 \mathrm{~J} / \mathrm{cm}^{2}$ with one $20 \mathrm{~ns}$ long pulse of a $\mathrm{KrF}$ excimer laser $(248 \mathrm{~nm})$ at J.P. Sercel Associates Inc. (New Hampshire, USA).

Depth profiles of Ti atoms in the Si lattice were obtained by time-of-flight secondary ion mass spectrometry (ToFSIMS) characterization. These were carried out with a TOFSIMS IV model manufactured by ION-TOF, using a $25 \mathrm{keV}$ pulsed $\mathrm{Bi}^{3+}$ beam at $45^{\circ}$ incidence. The secondary ions generated were extracted with a $10 \mathrm{keV}$ voltage and their time of flight from the sample to the detector was measured in a reflection mass spectrometer. Calibration was conducted using two reference unannealed samples implanted with two different energies and two different doses.

The structural characterization of the samples was carried out by glancing incidence $\mathrm{x}$-ray diffraction (GIXRD) and transmission electron microscopy (TEM). GIXRD patterns were obtained by a Panalytical X'Pert PRO MRD diffractometer working with a $\mathrm{Cu} K_{\alpha}$ source. The GIXRD patterns were collected at different incidence angles from $\omega$ $=0.1^{\circ}$ to $\omega=0.5^{\circ}$ to study the crystal structure of the samples at different depths. Cross sectional TEM images were obtained by a JEOL JEM-2000FX working at $200 \mathrm{keV}$. Simultaneously with the TEM measurements, electron diffraction (ED) patterns with a selected area of diffraction of about 50 $\mathrm{nm}$ were obtained. The ED images provided information on the crystalline morphology of the implanted layer. High resolution TEM (HRTEM) images were obtained by a JEOL JEM-4000 EX working at $400 \mathrm{keV}$.

Finally, we measured sheet resistance and Hall effect at variable temperature (90-400 K range) using a Keithley SCS 4200 model. The electromagnet field was fed with a bipolar Kepco BOP 50-20MG power supply. A detailed description of the experimental setup is given elsewhere. ${ }^{16}$

\section{RESULTS}

Figure 1 shows the Ti depth profiles obtained by ToFSIMS for the samples implanted with $10^{15}, 5 \times 10^{15}, 10^{16}$, and $5 \times 10^{16} \mathrm{~cm}^{-2}$ doses after a PLM process with an energy density of $0.8 \mathrm{~J} / \mathrm{cm}^{2}$. For clarity purposes, only the depth profiles of the reference as-implanted samples at $10^{15}$ and $10^{16} \mathrm{~cm}^{-2}$ has been added.

Comparing the profiles before and after the PLM we can observe a strong push effect toward the surface on the sample implanted with $10^{15} \mathrm{~cm}^{-2}$. The profile after PLM is now much sharper and the thickness that overcomes the Mott limit is clearly reduced to about $20 \mathrm{~nm}$. The summit of the Ti concentration profile reaches values close to $10^{21} \mathrm{~cm}^{-3}$, which is higher than the maximum of the as-implanted profile. Another maximum at a depth of about $50 \mathrm{~nm}$ can be observed. Note that this maximum is below the Mott limit and consequently should not form part of the IB material.

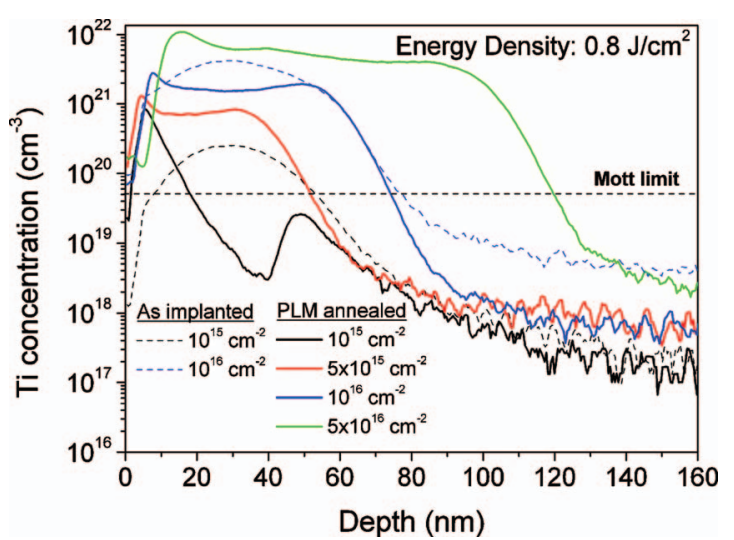

FIG. 1. (Color) ToF-SIMS profiles of Si samples implanted with Ti with doses of $10^{15}, 5 \times 10^{15}, 10^{16}$, and $5 \times 10^{16} \mathrm{~cm}^{-2}$ after PLM annealing at $0.8 \mathrm{~J} / \mathrm{cm}^{2}$. ToF-SIMS profiles of as-implanted samples with $10^{15}$ and $10^{16} \mathrm{~cm}^{-2}$ are also shown.

The push out effect is not so accentuated in the samples implanted with the higher doses, though all of them present profiles with a superficial Ti concentration peak after PLM. For all the doses presented here, the comparison between the integral of the profiles before and after PLM shows that about $40 \%$ of the Ti atoms have been lost during the annealing process at $0.8 \mathrm{~J} / \mathrm{cm}^{2}$.

Figure 2 shows the GIXRD pattern at $0.4^{\circ}$ glancing angle for the samples PLM annealed at $0.8 \mathrm{~J} / \mathrm{cm}^{2}$ and implanted with different doses. For the samples implanted with the highest doses $\left(10^{16}\right.$ and $\left.5 \times 10^{16} \mathrm{~cm}^{-2}\right)$, two diffraction peaks at $47.1^{\circ}$ and $55.9^{\circ}$ can be observed. For the sample with a dose of $5 \times 10^{15} \mathrm{~cm}^{-2}$, the peak placed at $47.1^{\circ}$ disappears, but the peak at $55.9^{\circ}$ is still slightly present. However, both diffraction peaks vanish for the sample implanted with the lowest dose $\left(10^{15} \mathrm{~cm}^{-2}\right)$. These peaks are attributed to the (220) and (113) silicon reflections. ${ }^{17}$ It is worth highlighting that no peaks associated to Ti-Si phases are present. These results point out the formation of a silicon polycrystalline structure for the samples implanted with the highest doses, without secondary $\mathrm{Ti}-\mathrm{Si}$ phases present in the implanted layer.

To further study the crystal structure at different depths, the GIXRD diffractograms were collected at different glancing angles. Figure 3 shows the GIXRD plot of the sample

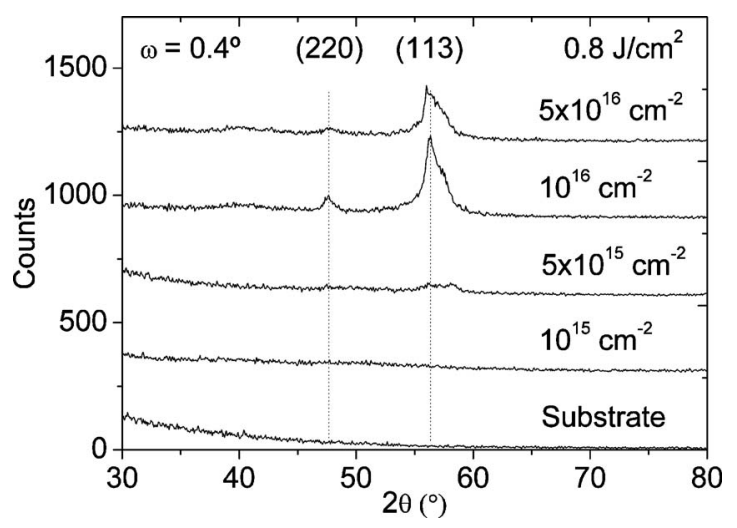

FIG. 2. GIXRD diffractograms at an incident angle of $0.4^{\circ}$ for the samples implanted with the different doses and annealed at $0.8 \mathrm{~J} / \mathrm{cm}^{2}$. 


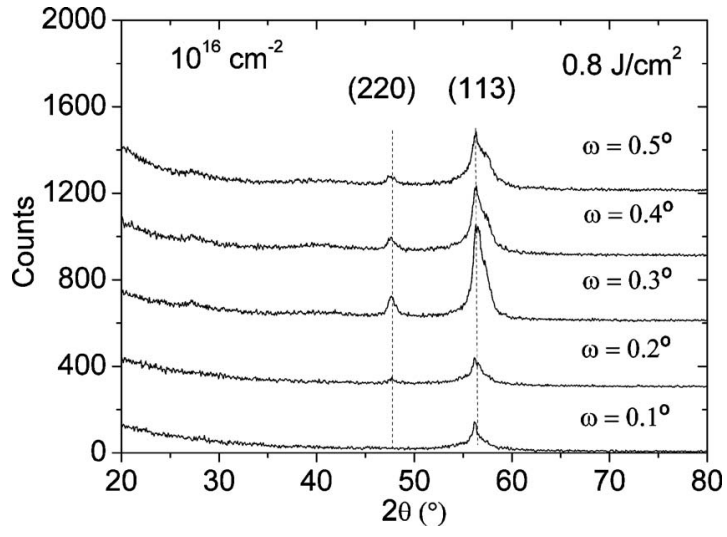

FIG. 3. GIXRD diffractograms at different incidence angles for the sample implanted with a $10^{16} \mathrm{~cm}^{-2}$ dose and annealed at $0.8 \mathrm{~J} / \mathrm{cm}^{2}$.

implanted with $10^{16} \mathrm{~cm}^{-2}$ and annealed at $0.8 \mathrm{~J} / \mathrm{cm}^{2}$. The intensities of the (220) and (113) silicon reflections increase with increasing the glancing incidence angle up to $0.3^{\circ}$, and decrease with higher incidence angles. Analogous behavior was observed for the sample implanted with the highest dose analyzed $\left(5 \times 10^{16} \mathrm{~cm}^{-2}\right)$. This trend hints again at the formation of a silicon polycrystalline phase in the implanted layer for the samples with the highest doses. However, no diffraction peaks were observed at any incidence angle for the sample implanted with the lowest dose $\left(10^{15} \mathrm{~cm}^{-2}\right)$, pointing in this case to a good recrystallization of the implanted layer. It is interesting to note that implantation with this dose $\left(10^{15} \mathrm{~cm}^{-2}\right)$ produces an extremely high Ti concentration, that exceeds in more than six orders of magnitude the solid solubility limit of $\mathrm{Ti}$ in $\mathrm{Si}^{18}{ }^{18}$ From these results, it is concluded that excellent recrystallization is obtained at the lowest implanted dose $\left(10^{15} \mathrm{~cm}^{-2}\right)$ and good recrystallization with the samples implanted with $5 \times 10^{15} \mathrm{~cm}^{-2}$. On the other hand, the samples implanted with $10^{16}$ and 5 $\times 10^{16} \mathrm{~cm}^{-2}$ showed poor recrystallization characteristics, remaining the implanted layer polycrystalline after the PLM process.

The definitive confirmation of differences in the crystalline structure among the samples is obtained through TEM images and ED measurements. Figure 4 shows the cross sectional TEM images and the ED patterns of samples implanted with different doses, and annealed at $0.8 \mathrm{~J} / \mathrm{cm}^{2}$. In the two first images, Fig. $4\left(\right.$ a) $\left(5 \times 10^{16} \mathrm{~cm}^{-2}\right.$ implantation dose) and Fig. 4(b) (10 ${ }^{16} \mathrm{~cm}^{-2}$ implantation dose), it can be observed a polycrystalline layer on top of a monocrystalline silicon substrate. The thicknesses of these polycrystalline layers were about $80 \mathrm{~nm}$ and $65 \mathrm{~nm}$, respectively. In the TEM images of Figs. 4(a) and 4(b), it is also appreciable the increase in grain size when the implanted dose decreases. The sample implanted with the highest dose [Fig. 4(a)] presents a nanocrystalline structure, with a mean grain size of about $5 \mathrm{~nm}$. When the implanted dose decreases, the grain size presents a dimension comparable to the implanted layer thickness. Regarding the ED patterns, Fig. 4(a) shows that the layer implanted with the highest dose $\left(5 \times 10^{16} \mathrm{~cm}^{-2}\right)$ is polycrystalline with small grains. This is indicated by the diffraction dots which are grouped in concentric rings around the direct beam. ED diagram of Fig. 4(b) (sample implanted

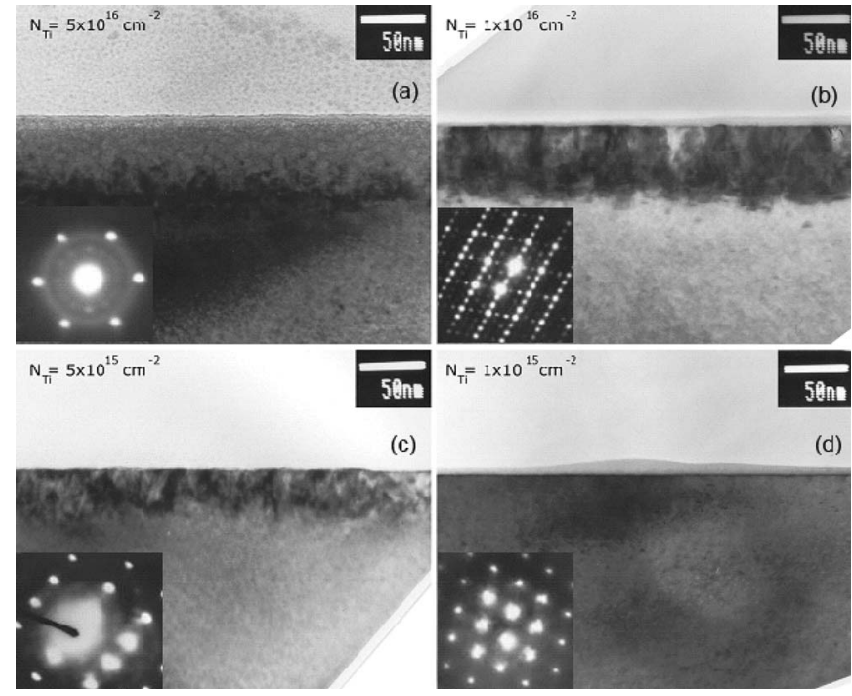

FIG. 4. Cross sectional TEM images and ED images of the samples implanted with different doses, and annealed at $0.8 \mathrm{~J} / \mathrm{cm}^{2}$ : (a) $5 \times 10^{16} \mathrm{~cm}^{-2}$, (b) $10^{16} \mathrm{~cm}^{-2}$, (c) $5 \times 10^{15} \mathrm{~cm}^{-2}$, and (d) $10^{15} \mathrm{~cm}^{-2}$.

with a $10^{16} \mathrm{~cm}^{-2}$ dose) presents an unusual pattern: the bright spots correspond to the [111] zone axes of the silicon, but intermediate spots appear between the main dots. This effect has been associated with the highly defective lattice reconstruction. ${ }^{19}$

Figures 4(c) and 4(d) show the TEM images of the samples implanted with the lowest doses. For the sample implanted with a dose of $5 \times 10^{15} \mathrm{~cm}^{-2}$ [Fig. 4(c)], the thickness of the defective implanted layer is reduced down to about $30-40 \mathrm{~nm}$ in some areas. For the sample implanted with a $10^{15} \mathrm{~cm}^{-2}$ dose [Fig. 4(d)], no contrast differences appear between the implanted layer and the substrate, thus, the defective layer is suppressed. In addition, the ED patterns of the implanted layer and the silicon substrate did not show difference, as it can be seen in Figs. 4(c) and 4(d). This result points to a good reconstruction of the lattice, and strengthens the GIXRD results.

Few areas with a different contrast were observed seldom-distributed in the implanted layer in Fig. 4(d). Figure 5(a) shows a HRTEM magnification of one of these defects and Fig. 5(b) the image of an of unimplanted unannealed silicon substrate. This different contrast in Fig. 5(a) is likely due to dangling bonds that produce stacking faults defects. Taking into account the high $\mathrm{Ti}$ concentration in the im-
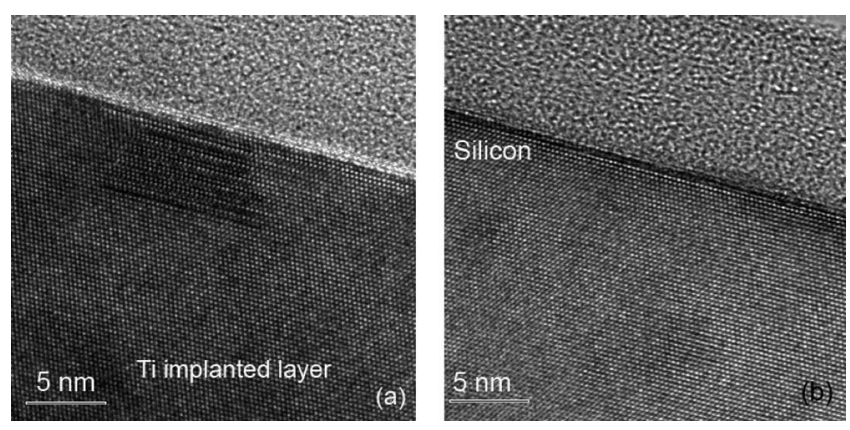

FIG. 5. HRTEM images of the sample implanted with a dose of $10^{15} \mathrm{~cm}^{-2}$, and PLM annealed at $0.8 \mathrm{~J} / \mathrm{cm}^{2}$ : (a) Ti-implanted layer and (b) Si substrate. 


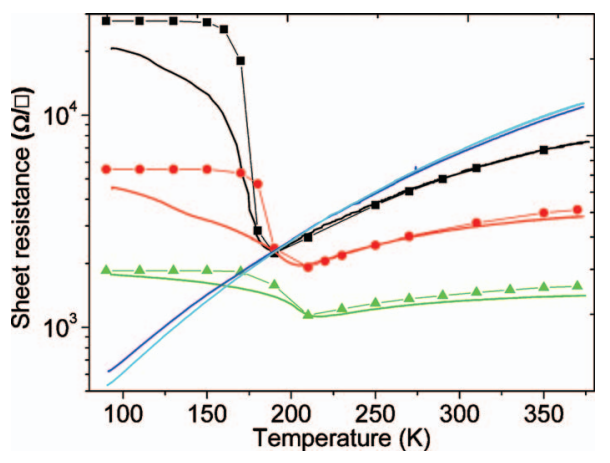

FIG. 6. (Color) Continuous lines are for measured sheet resistance of samples implanted with $10^{15} \mathrm{~cm}^{-2}(-), 5 \times 10^{15} \mathrm{~cm}^{-2}(-)$, and $10^{16} \mathrm{~cm}^{-2}(-)$ doses and PLM annealed with $0.8 \mathrm{~J} / \mathrm{cm}^{2}$, for a virgin substrate $(-)$ and for a PLM annealed but nonimplanted substrate $(-)$. Symbols are for ATLAS simulation results for samples implanted with $10^{15} \mathrm{~cm}^{-2}(\boldsymbol{\square}), 5 \times 10^{15} \mathrm{~cm}^{-2}(\boldsymbol{\bullet})$, and $10^{16} \mathrm{~cm}^{-2}(\boldsymbol{\Delta})$ doses and PLM annealed.

planted layer (about $10^{21} \mathrm{~cm}^{-3}$, i.e., a Ti concentration 6 orders of magnitude higher than the solid solubility limit), and the low density of these defects, this result points to an excellent crystalline reconstruction after the PLM process for this sample, as it was previously stressed by GIXRD data in Fig. 2. It should be remarked that with a $10^{15} \mathrm{~cm}^{-2}$ dose, we obtain an implanted layer with a Ti concentration higher than the Mott limit (see Fig. 1), with an almost perfect lattice reconstruction, i.e., a material of choice for IBSC. Nevertheless, it would be desirable a bulk IB material for a practical IBSC. More experiments are now being carried out to obtain thicker layers with similar quality.

Figure 6 presents the sheet resistance of the samples implanted with $10^{15}, 5 \times 10^{15}$, and $10^{16} \mathrm{~cm}^{-2}$ doses as a function of the measured temperature. In the same picture, we include the results of an ATLAS simulation taking into account the model explained in Ref. 16 that fits the IB location to $0.36 \mathrm{eV}$ down the conduction band, very close to one of the Ti experimental levels. ${ }^{20}$ Also we show the sheet resistance of a virgin and a nonimplanted but annealed substrate. The proposed model based on the IB formation has as main parameters the hole concentration at the IB, its mobility and the thickness of the Si region having this IB. Following the ToF-SIMS Ti profiles of Fig. 1, we have chosen $20 \mathrm{~nm}$ for the sample implanted with $10^{15} \mathrm{~cm}^{-2}$ dose, $60 \mathrm{~nm}$ for the sample implanted with $5 \times 10^{15} \mathrm{~cm}^{-2}$ dose and $80 \mathrm{~nm}$ for the sample implanted with $10^{16} \mathrm{~cm}^{-2}$ dose, obtaining a very good fitting for all the samples measured and improving the fittings previously reported in Ref. 21 where a single depth $(50 \mathrm{~nm})$ was estimated for all the doses.

The electrical model in Ref. 16 takes into account that we are dealing with a parallel device formed by the implanted layer and the substrate. This parallel behavior is clear at temperatures higher than the temperature of the sheet resistance minimum. Unexpectedly, at lower temperatures we observe a resistance higher than the one corresponding to the substrate, which is impossible in a parallel scheme. We explained this fact with a decoupling of the two layers due to the rectifying behavior of the junction that behaves as a nonideal junction. ${ }^{21}$ Note that this behavior would be impossible if the Ti level were a superficial level.
According to the model, the resistance at low temperatures is due just to the implanted layer because both layer are decoupled. The integration of the ToF-SIMS profiles leads to an effective dose of $60 \%$ of the implanted $\mathrm{Ti}$, meaning that the other $40 \%$ has been expelled during the PLM process, and according to this we can write, considering a constant depth distribution of $\mathrm{Ti}$ in the implanted layer: $\mathrm{R}_{\mathrm{s}}$ $=1 /\left(\mathrm{qp} \mu_{\mathrm{p}} \mathrm{t}\right)=1 /\left(\mathrm{q} 0.6 \mathrm{D} \mu_{\mathrm{p}}\right)$ where $\mathrm{p}$ is the hole concentration at the IB, $\mu_{\mathrm{p}}$ the mobility at this band and $\mathrm{D}$ is the dose. Although we obtain a direct relation between the amount of Ti impurities in the lattice and the sheet resistance of the implanted layer at low temperatures, we have to keep in mind that this is not the classical situation with a superficial dopant since only the interstitial Ti seems to be relevant for the formation of an IB. ${ }^{12}$ In this way, by means of Rutherford backscattering measurements, we have found that most of $\mathrm{Ti}$ is incorporated in interstitial locations after the implantation and PLM processes. ${ }^{22}$ The best fitting in the complete temperature range was obtained with $0.4 \mathrm{~cm}^{2} / \mathrm{V}$ s hole mobility for $10^{15}$ and $5 \times 10^{15} \mathrm{~cm}^{-2}$ doses and $0.6 \mathrm{~cm}^{2} / \mathrm{V} \mathrm{s}$ for $10^{16} \mathrm{~cm}^{-2}$ dose. The values of the estimated sheet resistances at low temperature are $26,5.2$, and $1.73 \mathrm{k} \Omega$ for the three doses. The congruence with the experimental data confirms the band formation by the totality of the Ti atoms which are distributed through the implanted thickness.

The higher mobility for the higher doped samples could be related with the expected wide energy thickness of the band when the Ti dose is increased. We have to keep in mind that, in general, the wider is a band, the higher is its mobility. This fact is also consistent with the higher carrier lifetime reported in Ref. 23 in spite of the high defect level of the samples implanted with $5 \times 10^{15}$ and $10^{16} \mathrm{~cm}^{-2}$ doses.

\section{CONCLUSIONS}

From GIXRD, TEM, and ED results, it can be concluded that the Si layer implanted with Ti with a dose of $10^{15} \mathrm{~cm}^{-2}$ and subsequently PLM annealed with an energy density of $0.8 \mathrm{~J} / \mathrm{cm}^{2}$ presents an excellent reconstruction of the crystal structure. The obtained $\mathrm{Ti}$ concentration reaches values as high as $10^{21} \mathrm{~cm}^{-3}$ without any significant silicon crystalline distortion. These results support a previous paper that showed that if $\mathrm{Ti}$ is incorporated at high concentration at interstitial positions it would form an IB band inside the $\mathrm{Si}$ gap [142]. ToF-SIMS profiles presented here confirm the impurity concentration required for the IB formation.

\section{ACKNOWLEDGMENTS}

Authors would like to acknowledge the Nanotechnology and Surface Analysis Services of the Universidad de Vigo C.A.C.T.I. for ToF-SIMS measurements, C.A.I. de Difracción de Rayos X of the Universidad Complutense de Madrid for GIXRD measurements, C.A.I. de Microscopía for TEM measurements and C.A.I. de Técnicas Físicas of the Universidad Complutense de Madrid for ion implantation experiments. This work was partially supported by the Projects NUMANCIA (Grant No. S-0505/ENE/000310) funded by the Comunidad de Madrid, GENESIS-FV (Grant No. CSD2006-00004) funded by the Spanish Consolider Na- 
tional Program, by the Grants (Grant No. CCG07-UCM/TIC2804) and (Grant No. GR58/08) funded by U.C.M.B.S.C.H., and by the Project NUMANCIA-2 (Grant No. S2009/ENE/1477) funded by the Comunidad de Madrid.

${ }^{1}$ A. Martí and A. Luque, Next Generation Photovoltaics: High Efficiency through Full Spectrum Utilization (Institute of Physics, Bristol, UK, 2004).

${ }^{2}$ A. Luque and A. Martí, Phys. Rev. Lett. 78, 5014 (1997).

${ }^{3}$ M. A. Green, Third Generation of Photovoltaics (Springer, Berlin, Germany, 2005).

${ }^{4}$ W. Shockley and H. J. Queisser, J. Appl. Phys. 32, 510 (1961).

${ }^{5}$ A. Martí, E. Antolin, C. R. Stanley, C. D. Farmer, N. López, P. Díaz, E. Cánovas, P. G. Linares, and A. Luque, Phys. Rev. Lett. 97, 247701 (2006).

${ }^{6}$ A. Luque, A. Marti, N. Lopez, E. Antolin, E. Canovas, C. Stanley, C. Farmer, L. J. Caballero, L. Cuadra, and J. L. Balenzategui, Appl. Phys Lett. 87, 083505 (2005).

${ }^{7}$ K. M. Yu, M. A. Scarpulla, R. Farshchi, O. D. Dubon, and W. Waluckievicz, Nucl. Instrum. Methods Phys. Res. B 261, 1150 (2007).

${ }^{8}$ N. F. Mott, Rev. Mod. Phys. 40, 677 (1968).

${ }^{9}$ K. M. Yu, W. Walukiewicz, J. W. Ager III, D. Bour, R. Farshchi, O. D. Doubon, S. X. Li, D. Sharp, and E. E. Haller, Appl. Phys. Lett. 88, 092110 (2006).

${ }^{10}$ W. Shan, W. Walukiewicz, J. W. Ager III, E. E. Haller, J. F. Geisz, D. J. Friedman, J. M. Olson, and S. R. Kurtz, Phys. Rev. Lett. 82, 1221 (1999).
${ }^{11}$ K. M. Yu, W. Walukiewicz, J. Wu, W. Shan, J. W. Beeman, M. A. Scarpulla, O. D. Dubon, and P. Becla, Phys. Rev. Lett. 91, 246403 (2003).

${ }^{12}$ K. Sánchez, I. Aguilera, P. Palacios, and P. Wahnón, Phys. Rev. B 79, 165203 (2009).

${ }^{13}$ J. Olea, M. Toledano-Luque, D. Pastor, G. González-Díaz, and I. Mártil, J. Appl. Phys. 104, 016105 (2008).

${ }^{14}$ P. Palacios, J. J. Fernández, K. Sánchez, J. C. Conesa, and P. Wahnón, Phys. Rev. B 73, 085206 (2006).

${ }^{15}$ M. Tabbal, T. Kim, J. M. Warrender, M. J. Aziz, B. L. Cardozo, and R. S. Goldman, J. Vac. Sci. Technol. B 25, 1847 (2007).

${ }^{16}$ J. Olea, G. González-Díaz, D. Pastor, and I. Mártil, J. Phys. D: Appl. Phys 42, 085110 (2009).

${ }^{17}$ B. D. Cullity and S. R. Stock, Elements of X-Ray Diffraction, 3rd ed. (Prentice-Hall, Massachusetts, 2001).

${ }^{18}$ S. Hocine and D. Mathiot, Appl. Phys. Lett. 53, 1269 (1988).

${ }^{19}$ D. B. Williams and B. C. Carter, Transmission Electron Microscopy: Diffraction, (Plenum, New York, USA, 1996), Vol. 2.

${ }^{20}$ D. Mathiot and S. Hocine, J. Appl. Phys. 66, 5862 (1989).

${ }^{21}$ G. Gonzalez-Díaz, J. Olea, I. Mártil, D. Pastor, A. Martí, E. Antolín, and A. Luque, Sol. Energy Mater. Sol. Cells 93, 1668 (2009).

${ }^{22}$ J. Olea, D. Pastor, I. Mártil, G. González-Díaz, J. Ibáñez, R. Cuscó, and L. Artús, Proceedings of the 2009 Materials Research Society Fall Meeting, Boston (USA), 2009 (unpublished).

${ }^{23}$ E. Antolín, A. Martí, J. Olea, D. Pastor, G. González-Díaz, I. Mártil, and A. Luque, Appl. Phys. Lett. 94, 042115 (2009). 